\title{
Stable plasma configurations in a cylindrical magnetron discharge
}

\author{
I. Levchenko ${ }^{\text {a) }}$ and M. Romanov \\ National Aerospace University, Kharkov 61003, Ukraine \\ M. Keidar \\ Department of Aerospace Engineering, The University of Michigan, Ann Arbor, Michigan 48109 \\ I. I. Beilis \\ Electrical Discharge and plasma Laboratory, Tel Aviv University, Tel Aviv, P.O.B. 39040, \\ Tel Aviv 69978, Israel
}

(Received 26 April 2004; accepted 14 July 2004)

\begin{abstract}
Transition between different plasma configurations is studied in a system with negative biased cylindrical target in crossed $E \times B$ fields. It was found that the diffuse plasma torus formed around the cylindrical target in relatively small magnetic field $(0.02 \mathrm{~T}$ on target surface) changes the shape with magnetic field to form a thin disk with a width lower than $1 \mathrm{~cm}$ when target voltage is less than $-400 \mathrm{~V}$. The target current decreases sharply when the magnetic field reaches some critical value. When the target voltage exceeds $400 \mathrm{~V}$, the target current increases with the magnetic field and the plasma has always toroidal shape. The plasma behavior can be understood taking in account the interaction of the drift currents and the magnetic field. (C) 2004 American Institute of Physics.
\end{abstract}

[DOI: 10.1063/1.1792795]

Magnetron discharges have various applications for thin film deposition, etching, etc. ${ }^{1}$ In addition, some promising results were obtained using magnetron discharge for lowvoltage plasma immersion. ${ }^{2-5}$ Use of the magnetic field in the plasma immersion implantation system provides appreciable advantages, in comparison with conventional method. ${ }^{6,7}$ The magnetic field influences significantly the sheath thickness in plasma immersed ion implantation process. ${ }^{8}$ Previously both planar and cylindrical magnetron discharge geometries were considered, however transition between various plasma configurations in magnetron system was not studied in detail. In this work we present experimental study of two stable plasma configurations in a cylindrical magnetron discharge.

A scheme of experimental setup is shown in Fig. 1. We used a system with crossed $E \times B$ fields similar to that used in cylindrical magnetrons. ${ }^{9}$ The system was installed in a vacuum chamber with a volume of $0.25 \mathrm{~m}^{3}$ equipped with vacuum pump and gas-supplying system, capable of maintaining a nitrogen pressure of $0.1-1.0 \mathrm{~Pa}$. Grounded walls of the vacuum chamber were located $25 \mathrm{~cm}$ from the cylindrical target. A tubular negatively biased target (ion collecting electrode) with a length of $500 \mathrm{~mm}$ and diameter of $120 \mathrm{~mm}$ made of nonmagnetic stainless steel was placed into the axial magnetic field created by a set of coils. This way the main component of magnetic field vector is parallel to the target surface, and intersects the metal surface at target edges. With target biased, the crossed system of radial electrical field and longitudinal magnetic field is created, providing electron drift around the target as it takes place in Hall thrusters. ${ }^{10}$

The magnetic field strength was controlled by changing the coil current. The screens were installed on the ceramic rings, so the screens were placed under the floating plasma potential to prevent arcing on the target edges. The power supply unit used in this experiment was capable of providing the bias voltage from 0 to $2 \mathrm{kV}$. The plasma parameters were

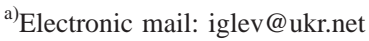

measured with Langmuir probes connected to the automatic data acquisition system with the time constant of about $100 \mu \mathrm{s}$.

The intense ionization of a background gas in crossed $E \times B$ field system near the biased target leads to the bright plasma torus formation around the cylindrical target [see Fig. 2(a)]. The detailed description of this system, magnetic field configuration and parameters was described elsewhere. ${ }^{4}$

Increase of magnetic field strength in this system has a complicated affect on the plasma formed around the target surface. When bias voltage exceeds $-400 \mathrm{~V}$, the target current increases with the magnetic coil current (and hence the magnetic field strength), and the higher target current values correspond to the higher pressures, as shown in Fig. 3(a). This mode corresponds to the bright plasma torus shown in

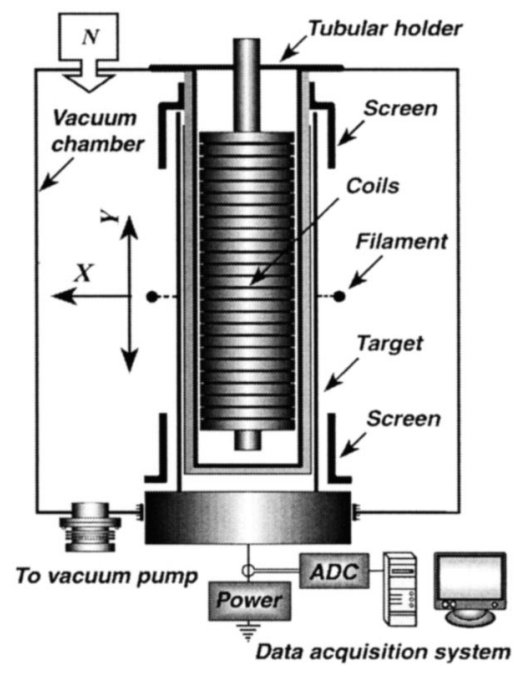

FIG. 1. Experimental setup.

Fig. 2(a), and is characterized by high target currents reach- 
(a)
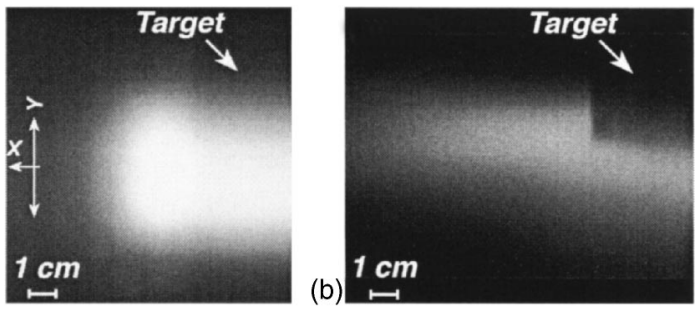

FIG. 2. Photograph of plasma in torus mode (a) and disk mode (b).

ing several amperes, which corresponds to the current density of about $500 \mathrm{~A} / \mathrm{m}^{2}$.

When the bias voltage is less than $-400 \mathrm{~V}$, plasma behavior changes. The increase of target current occurs at lower magnetic fields, when the coil current does not exceed $2 \ldots 3 \mathrm{~A}$. Then with the magnetic field increasing, a weak decrease in target current takes place. And finally, when the magnetic field approaches some critical value, corresponding to the coil current of $8 \mathrm{~A}$ approximately in this setup, the target current falls down sharply to the value of about 50-100 mA. Simultaneously, plasma configuration changes and takes the shape of a narrow disk with a width of approximately $1 \mathrm{~cm}$ and large diameter equal to the maximum diameter of the primary torus. The general appearance of the plasma disk is shown in Fig. 2(b), and the current drop is shown in Fig. 3(b). A subsequent increase of magnetic field strength leads to slow decrease of target current and plasma brightness, without any transitions and additional effects.

The disk mode has several features that were clearly noted in the experiments. The first feature is the abrupt character of transition to the disk. We changed the coil current with steps of $0.1 \mathrm{~A}$; it was noted that the complete change to the disk occurred within the single current step, whereas these steps could provide only insignificant changes in the plasma torus shape and brightness before transition. Similarly, the following change of coil current after disk formation leads to only slight change in disk shape and brightness.

The second feature is the strong change of plasma diameter/thickness ratio, as compared with the primary plasma torus. The decrease of plasma thickness happens within one step of coil current $(0.1 \mathrm{~A})$, and no stable intermediate shapes were noticed. It should be noted that the plasma torus decreases slightly when approaching the torusto-disk transition critical current (8 A approximately), but this decrease is small relative to the primary torus size, and the transition to disk mode occurs always abruptly. As a rule, the plasma thickness decreases in jump from $3-4 \mathrm{~cm}$ to $1 \mathrm{~cm}$ or less, i.e., thrice at least. Besides, several oscillations may be sometimes observed after transition to the disk, if the critical magnetic field is being adjusted smoothly. During
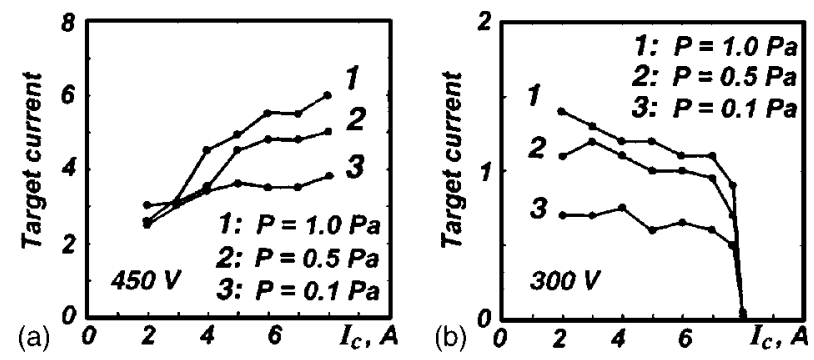

FIG. 3. Target current vs coil current with pressure as a parameter.

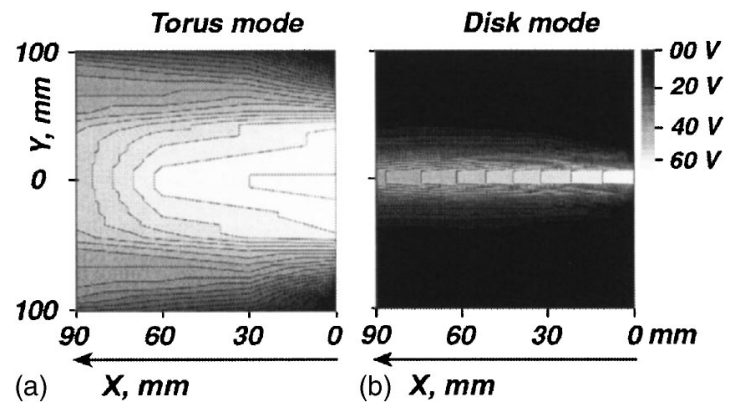

FIG. 4. Plasma potential fields.

these oscillations, plasma sharply changes the shape from the torus to disk and back to the torus with the frequency of $1 \mathrm{~Hz}$ approximately. To stabilize the state, slight change of magnetic field strength is required in this case.

A spatial distribution of plasma parameters in both torus and disk modes was investigated using a set of Langmuir probes. We used ten probes made of wire of $2 \mathrm{~mm}$ in diameter covered with ceramic insulation. The distance between probes was 5-10 $\mathrm{mm}$, so the probes covered a length of $100 \mathrm{~mm}$ at once. A special gear provided movement of the probes along plasma torus/disk axis, and turning the whole probe set around the own axis, to provide twofold coverage of $200 \mathrm{~mm}$.

The results of probe measurements are shown in Figs. 4(a) and 4(b). It is evident that the probe studies conform to the visual photographs shown in Figs. 2(a) and 2(b). In the disk mode [see Fig. 4(b)] the plasma is located in the narrow area along the axis, in contrast to plasma torus [Fig. 4(a)] when the plasma is distributed in a wide area. Besides, one more feature should be mentioned here, namely relatively high plasma potential in the disk mode. It can be seen that during transition from plasma torus to plasma disk the plasma potential decreases in the areas remote from the disk axis, and does not change in the areas located near the axis. The measurements of electron temperature provided the maximum values of about $6 \mathrm{eV}$ for the plasma located near the disk and torus axis. The spatial distribution of the electron temperature is similar to the plasma potential distribution.

Since the magnetic field and plasma structures are highly nonuniform, a complete quantitative model of this system could be very complicated and goes beyond the scope of this letter. In this work we present a qualitative model with an aim to capture leading processes that determine stable plasma configurations and their behavior.

The most striking feature of the observed phenomena is the dramatic decrease of the target current in disk mode as compared with the torus mode. Let us start from analysis of the diffuse torus plasma mode [Fig. 2(a)]. When bias voltage is high, the stable plasma discharge can be created. Some features of this type of discharge were described previously. ${ }^{11,12}$ Let us assume that almost all of the applied voltage is dropped across the near target sheath. In this case the ions bombarding the target gain energy $e U_{b}$. The steady plasma discharge is sustained by primary electrons that originate from ion secondary emission from the target. Electrons gain the energy in the sheath and the condition for required bias voltage to sustain the steady state discharge can be roughly estimated as follows (Ref. 11): 


$$
U_{b} \gamma_{i}=\varepsilon_{c}+\frac{E_{\mathrm{loss}}}{\tau_{e} \nu_{i z}},
$$

where $\gamma_{i}$ is the ion secondary emission coefficient, $\boldsymbol{\varepsilon}_{c}$ is energy lost per electron-ion pair created by the primary electrons, eV; $E_{\text {loss }}$ is energy losses (to the walls, collisions with gas, electron-electron collisions and others, Ref. 11), eV; $\tau_{e}$ is the electron residence time, $\mathrm{s} ; \nu_{i z}$ is the ionization frequency, $\mathrm{s}^{-1}$. It is known ${ }^{13,14}$ that ion electron emission yield in the low ion energy range $(<1 \mathrm{kV})$ has very weak dependence on the ion energy. In our conditions ( $N$ ions and $\mathrm{Fe}$ target) the ion electron emission coefficient is about $\gamma_{i}$ $=0.096$. Taking into account that the energy loss per electron-ion pair created by the primary electrons $\varepsilon_{c}$ is about $30 \mathrm{eV}$ (Ref. 11) and accounting for losses to the chamber wall and collisions with gas, one can find that the necessary voltage drop is about $-300-400 \mathrm{~V}$. This number is close to that observed in our experiment $(\sim 400 \mathrm{~V})$ as a necessary condition for diffuse torus mode existence. Another condition for the stable configuration is that the particle losses to the chamber wall should be balanced by the volume ionization. In the torus mode the increase of the magnetic field leads to additional ionization due to increased electron residence time (due to closed electron drift) and decreased wall losses due to suppression of the electron mobility across the magnetic field. Therefore one can expect that the target current would increase with magnetic field in agreement with experimental observation [torus mode, see Fig. 3(a)].

On the other hand one can see that in the low-voltage disk mode [Fig. 2(b)], $U_{b}$ is small to sustain the discharge [according to Eq. (1)]. It is possible that the electron impact ionization (which is the main source of cold electrons with energy 3-5 eV) plays an essential role in the discharge stability in this mode. The relatively low electron impact ionization rate is enhanced in the central zone where the magnetic field lines are parallel to the target surface and strictly orthogonal to the electrical field lines. In this area the electron magnetization and the closed electron drift associated with it are strongest. Magnetized electrons that originated at the target surface diffuse across the magnetic field due to rare collisions and provide volume gas ionization. On the other hand, the electrons from peripheral areas escape the magnetized region and are lost to walls. In order to keep particle balance, the diffusion time should be comparable to the ionization time:

$$
\frac{1}{\nu_{i z}} \cong \frac{L}{\mu_{B} E},
$$

where $L$ is the characteristic size of the system $\mu_{B}$ $=(16 B)^{-1}$ is the Bohm mobility, and $E$ is the electrical field [can be estimated from our measurements, Fig. 4(b), $\left.\sim 10^{3} \mathrm{~V} / \mathrm{m}\right]$. Taking the ionization collision frequency in the form: $\nu_{i z}=\nu_{e} \sigma_{i z} n$, where $\sigma_{i z}$ is the ionization collision cross section, one can obtain the relation for the magnetic field:

$$
B=\frac{E}{16 L V_{e} \sigma_{i z} n},
$$

where $n$ is the background gas density. The ionization cross section for nitrogen and low-energy electrons has an order of $10^{-21} \mathrm{~m}^{2}$. Assuming $L=0.1 \mathrm{~m}, n=5 \times 10^{19} \mathrm{~m}^{-3}$, and electron temperature of about $5 \mathrm{eV}$, we assess the critical magnetic field of about $B=0.01 \mathrm{~T}$, which corresponds to the coil current of about $I_{c}=7 \mathrm{~A}$ and distance from target of $0.1 \mathrm{~m}$. Therefore at higher magnetic field strength the diffusion across the magnetic field from the target surface to periphery is suppressed and discharge cannot be sustained. An estimated value of the magnetic field is close to that found in our experiment as shown in Fig. 3(b).

To check the above-mentioned conclusion about leading role of the electron density and electron current in plasma behavior, we have made an additional experiment with an incandescent filament installed just above the plasma plane of symmetry (shown in Fig. 1). This experiment had showed that an additional electron emission from the filament causes immediate transition from disk mode to plasma torus mode. Besides we have found that when incandescent filament is used, the torus may be formed at lower voltage and lower magnetic field, i.e., in conditions when discharge was absent without filament. All the above said proves our conclusion that disk mode is formed when the electron current cannot sustain the total current due to low mobility in magnetic field, or due to low electron density in the conditions when ionization is too weak.

In summary, we have experimentally found that the plasma behavior in a system with crossed electrical and magnetic fields depends strongly on the magnetic field strength. With the magnetic field approaching $0.1 \mathrm{~T}$ at the target surface, the current decreases from several amperes to several tenths of milliamperes. This process is accompanied by essential change of plasma shape, and the plasma reshapes to a thin disk. The results are explained in terms of ionization by magnetized electrons. The results may be important for understanding plasma behavior in technological systems like cylindrical magnetrons, and in Hall thrusters.

The authors acknowledge Dr. Yevgeny Raitses for fruitful discussions.

\footnotetext{
${ }^{1}$ S. M. Rossnagel, J. Vac. Sci. Technol. A 6, 19 (1988).

${ }^{2}$ Handbook of Plasma Immersion Ion Implanatation and Deposition, edited by A. Anders (Wiley, New York, 2000).

${ }^{3}$ A. Anders, Surf. Coat. Technol. 93, 157 (1997).

${ }^{4}$ I. Levchenko, M. Romanov, and M. Keidar. J. Appl. Phys. 94, 1408 (2003).

${ }^{5}$ I. Levchenko, M. Romanov, and M. Korobov, Surf. Coat. Technol. 184, 356 (2004).

${ }^{6}$ A. Loinaz, M. Rinner, F. Alonso, J. I. Onate, and W. Ensinger., Surf. Coat. Technol. 103-104, 262 (1998).

${ }^{7}$ I. Levchenko, M. Romanov, O. Baranov, and M. Keidar, Vacuum 72/3, 335 (2003).

${ }^{8}$ M. Keidar, O. R. Monteiro, A. Anders, and I. D. Boyd., Appl. Phys. Lett. 81, 1183 (2002).

${ }^{9}$ A. Yonesu, H. Takemoto, M. Hirata, and Y. Yamashiro. Vacuum 66, 275 (2002).

${ }^{10}$ M. Keidar, I. D. Boyd, and I. I. Beilis., Phys. Plasmas 8, 5315 (2001).

${ }^{11}$ Z. Wang and S. A. Cohen, J. Vac. Sci. Technol. A 17, 77 (1999).

${ }^{12}$ Z. Wang and S. A. Cohen, Phys. Plasmas 6, 1655 (1999).

${ }^{13}$ Yu. P. Raizer, Gas Discharge Physics (Springer, New York, 1991).

${ }^{14}$ M. A. Lieberman and A. J. Lichtenberg, Priniciples of Plasma Discharges and Material Processing (Wiley, New York, 1994).
} 